\title{
Nanoparticles and Nanopores Induced by High-energy Electron Irradiation
}

\author{
Nan Jiang*, Dong Su** and John C. H. Spence* \\ * Department of Physics, Arizona State University, Tempe, AZ 98287 \\ ** Center for Functional Nanomaterials, Brookhaven National Laboratory
}

Nanoparticles and nanopores inside materials have a wide range of applications. There are various methods available to precipitate nanoparticles and to produce nanopores inside materials, such as thermal annealing and deposition. Here we will focus on using high-energy electron-beam irradiation in a transmission electron microscopy (TEM). The main advantage of this method is that the precipitation can be confined within a desired area, and thus it allows direct-write lithography. Two examples will be discussed in this paper: one is the precipitation of $\mathrm{Na}$ nanoparticles in femtosecond (fs) laser pulses pre-irradiated soda-lime silicate glass, and the other is the formation of nanopores in $\mathrm{MgO}$ by high-energy electron beams.

As we knew, $\mathrm{Na}$ in silicate glasses is very unstable under electron beams. Although $\mathrm{Na}$ can be neutralized easily under electron beams by capturing electrons which are released from atoms by ionization and the Auger process, it can be also re-oxidized quickly by oxygen ions which are released from the glass matrix by electron excitations [1]. Therefore, the well-shaped $\mathrm{Na}$ nanoparticles cannot be precipitated in the normal glasses by electron-beam irradiation. However, the Na nanoparticles can be precipitated from the glasses which were pre-treated by fs-laser pulses. As shown in Fig. 1 (a) and (b), the Na particles with relatively uniform shape can be produced in the soda lime silicate glass under electron beam irradiation. As shown in Fig. 1(c), the comparison of EELS spectra recorded on particle and on matrix confirms that these particles are Na. Most likely, the fs-laser irradiation creates the "embryos" of Na clusters, which are immobile under electron beam. The growth of these "embryos", however, can be fulfilled under electron irradiation.

Nanoporous $\mathrm{MgO}$ can be formed by the thermal dehydration process of $\mathrm{Mg}(\mathrm{OH})_{2}$, as well as by electron-beam irradiation. In our previous study, it reported that the dehydration process by e-beam is in fact different from that by the thermal annealing [2]. Furthermore, the produced porous $\mathrm{MgO}$ are also slightly different between these two methods. As compared in the bright filed (BF) images in Fig. 2, the porous $\mathrm{MgO}$ by the e-beam irradiation has finer morphology than that by the thermal annealing. We suggest that the nanopores in e-beam produced porous $\mathrm{MgO}$ are smaller than those in the thermally annealed sample. Although it is difficult to quantify the size of nanopores from both the diffraction- and phase-contrast images, the porosity can be evaluated by the valence electron energy-loss spectroscopy [3]. As shown in Fig. 3, the maximum peak position in valence EELS is slightly lower in the e-beam induced than that in the thermally induced porous $\mathrm{MgO}$. The difference can be interpreted as the result of different porosity in these samples. The method is based on the effective medium dielectric theory [4].

References

[1] N. Jiang, D. Su, and J. C. H. Spence, Appl. Phys. Lett. 91, 231906 (2007).

[2] D. Su, N. Jiang, J. C. H. Spence, F. He, and W. T. Petuskey, J. Appl. Phys. 104, 063514 (2008).

[3] N. Jiang, D. Su, A. Howie, and J. C. H. Spence, in preparation.

[4] A. Howie and C. Walsh, Microsc. Microanal. Microstruct. 2, 171 (1991); references therein. 
[5] This work is supported by NSF DMR0603993. The use of facilities within the center for solid state science at ASU is also acknowledged.
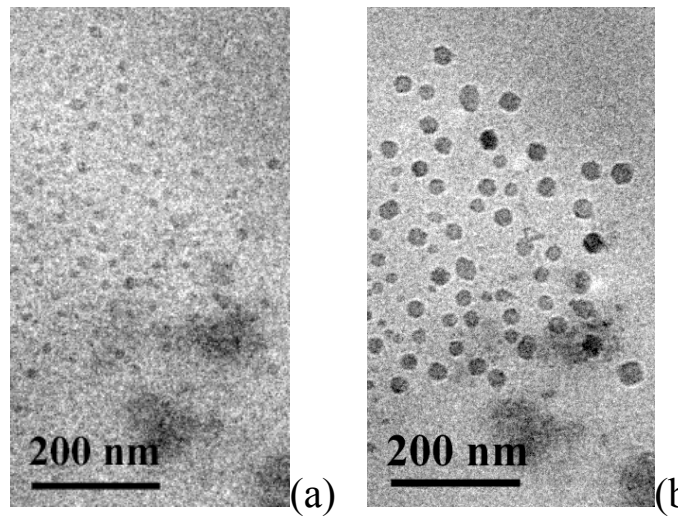

(a)

(b)

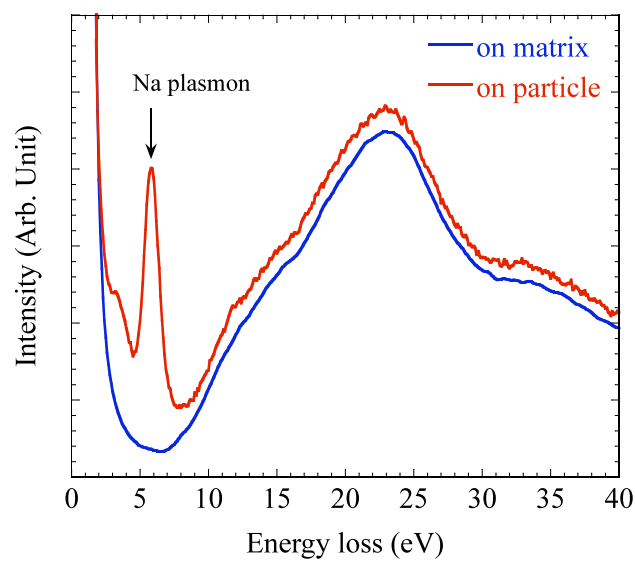

(c)

Figure $1 \mathrm{BF}$ images showing the growth of Na nanoparticles by electron beams in the fs-laser preirradiated soda lime silicate glass. The images were taken (a) right after the area exposed to electron beam, (b) after several minutes. (c) EELS spectra recorded on nanoparticle and on glass matrix.

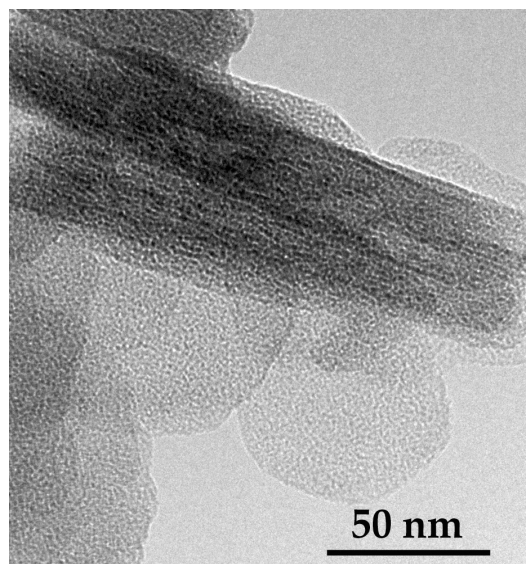

(a)

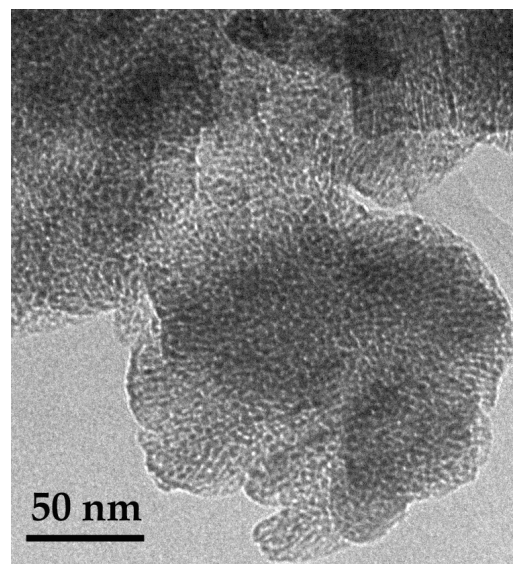

(b)

Figure 2 TEM BF images showing porous $\mathrm{MgO}$ induced by (a) e-beam and (b) thermal annealing.

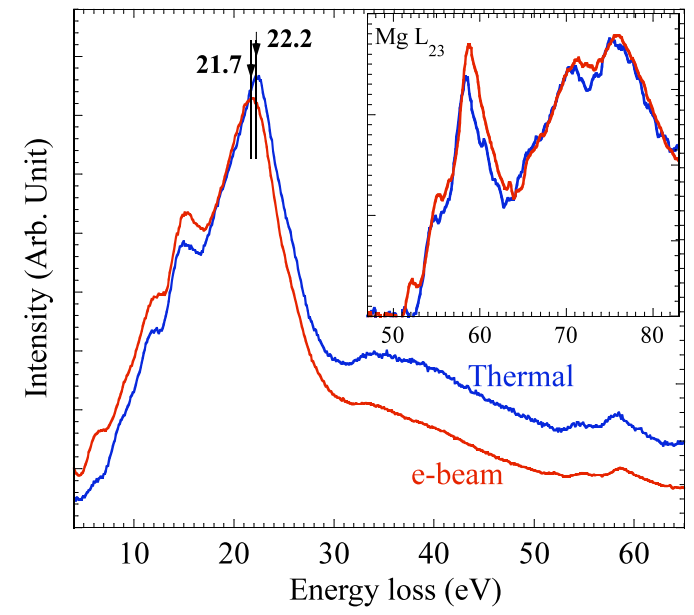

Figure 3 Comparison of EELS recorded in the porous $\mathrm{MgO}$ produced by e-beam and thermal annealing. 